\title{
Timestepping schemes for the 3d Navier-Stokes equations
}

\author{
Youngjoon Honga, ${ }^{a, *}$ Djoko Wirosoetisno ${ }^{\mathrm{b}}$ \\ ${ }^{a}$ Department of Mathematics, Indiana University, Bloomington, IN 47405-7106, United \\ States \\ ${ }^{b}$ Mathematical Sciences, Durham University, Durham DH1 3LE, United Kingdom
}

\begin{abstract}
It is well known that the (exact) solutions of the 3d Navier-Stokes equations remain bounded for all time if the initial data and the forcing are sufficiently small relative to the viscosity. They also remain bounded for a finite time and for an arbitrary initial data in $L^{2}$. In this article, we consider two temporal discretisations (semi-implicit and fully implicit) of the 3d Navier-Stokes equations in a periodic domain and prove that their solutions remain uniformly bounded in $H^{1}$ subject to essentially the same respective smallness conditions as the continuous system (on initial data and forcing or on the time of existence) provided the time step is small.
\end{abstract}

Keywords: 3d Navier-Stokes, small solutions, short time, temporal discretisation, Euler schemes

2010 MSC: 65M12, 76D05

\section{Introduction}

Much work has been done on the stability and convergence of various timestepping schemes for the Navier-Stokes equations in two space dimensions (2d NSE). The long time stability in $H^{1}$ of the implicit Euler scheme for the 2d NSE has

5 been treated in, e.g., [2, 6, 4, 8], and more recently extended to higher-order

\footnotetext{
* Corresponding author

Email address: hongy@indiana.edu (Youngjoon Hong)
}

Preprint submitted to Applied numerical mathematics

April 17, 2015

(C) 2015. This manuscript version is made available under the Elsevier user license http://www.elsevier.com/open-access/userlicense/1.0/ 
schemes in [9, 3]. Once the numerical solutions are shown to be bounded in suitable space, either on a limited interval of time or for all time, convergence can usually be established using standard techniques (cf., e.g., [5]).

In three dimensions, the existence of a solution bounded in $L^{\infty}\left(\mathbb{R}_{+}, H^{1}\right)$ is not known; we only know the existence of a globally bounded solution if the data are small and of a locally bounded solution for data of arbitrary size; for more background on the NSE, see e.g. [1, 7]. Hence, the extension of the numerical stability results from $2 \mathrm{~d}$ or $3 \mathrm{~d}$ is not straightforward. We conduct it here in the two cases for which the existence of a strong solution is known, namely, as we said, a globally bounded solution associated with small data or a locally bounded solution associated with arbitrary data.

In this article we consider temporal discretisations of the $3 \mathrm{~d}$ NSE using a semi-implicit Euler scheme and the fully implicit Euler scheme; see (2.1) and (3.1) below. For both of these schemes, we establish the uniform boundedness in $H^{1}$ for the discrete solutions for interval of times corresponding to the existence of solutions. As in the earlier works cited above, we do not consider time and space discretisations, giving the advantage that our results will be free of Courant-Friedrichs-Lewy-type constraints, although some smallness of the timestep is required.

We consider the Navier-Stokes equations in $\Omega=(0,2 \pi)^{3}$ with periodic boundary conditions,

$$
\begin{aligned}
& \partial_{t} u+(u \cdot \nabla) u+\nabla p=\nu \Delta u+f, \\
& \nabla \cdot u=0
\end{aligned}
$$

plus the initial data $u(0)=u_{0}$. With no loss of generality, we assume that $\nabla \cdot f=0$, and that the integrals of $f$ and $u_{0}$ vanish over $\Omega$. The last assumption implies that $u=u(t)$, whenever it is well-defined for $t \geq 0$, also has vanishing integral over $\Omega$, giving us the Poincaré inequality

$$
|u|_{L^{2}}^{2} \leq c_{0}(\Omega)|\nabla u|_{L^{2}}^{2}
$$

For notational convenience, we redefine $c_{0}$ to give also the bound (for $u$ periodic 
with average 0$)$ :

$$
|\nabla u|_{L^{2}}^{2} \leq c_{0}|\Delta u|_{L^{2}}^{2}
$$

In order to facilitate comparison with the numerical solutions, in the rest of this section we briefly review the boundedness of solutions of the 3d NSE, both 35 in $L^{2}$ and in $H^{1}$ for the two cases (small data, large time and short time for arbitrary data).

Multiplying (1.1) by $u$ in $L^{2}(\Omega)$, integrating by parts and using the fact that $((u \cdot \nabla) u, u)=0$, we find

$$
\frac{1}{2} \frac{\mathrm{d}}{\mathrm{d} t}|u|^{2}+\nu|\nabla u|^{2}=(f, u) .
$$

Here and henceforth, unadorned norm $|\cdot|$ and inner product $(\cdot, \cdot)$ are taken

to be $L^{2}$. Bounding the rhs by the Cauchy-Schwarz inequality and using the Poincaré inequality, (1.4) becomes

$$
\frac{\mathrm{d}}{\mathrm{d} t}|u|^{2}+\frac{\nu}{c_{0}}|u|^{2} \leq \frac{1}{\nu}|f|_{L^{\infty}\left(H^{-1}\right)}^{2},
$$

where $|f|_{L^{\infty}\left(H^{-1}\right)}:=\sup _{t \geq 0}|f(t)|_{H^{-1}}$. Hence, we have

$$
\frac{\mathrm{d}}{\mathrm{d} t}\left(|u|^{2} \exp \left(\frac{\nu}{c_{0}} t\right)\right) \leq \exp \left(\frac{\nu}{c_{0}} t\right) \frac{1}{\nu}|f|_{L^{\infty}\left(H^{-1}\right)}^{2} .
$$

Integrating from 0 to $t$ (here we change the dummy variable of integration to $s$ ), we obtain

$$
|u(t)|^{2} \exp \left(\frac{\nu}{c_{0}} t\right) \leq|u(0)|^{2}+\frac{c_{0}}{\nu^{2}}|f|_{L^{\infty}\left(H^{-1}\right)}^{2}\left(\exp \left(\frac{\nu}{c_{0}} t\right)-1\right) .
$$

45 We then find the uniform $L^{2}$ bound valid for all $t \geq 0$ :

$$
|u(t)|^{2} \leq|u(0)|^{2}+\left(c_{0} / \nu^{2}\right)|f|_{L^{\infty}\left(H^{-1}\right)}^{2}=: K_{0}\left(u_{0}, f ; \nu, \Omega\right) .
$$

\section{1. $H^{1}$ estimate for small data}

Now multiplying (1.1) by $-\Delta u$ in $L^{2}(\Omega)$ and integrating by parts, we find

$$
\frac{1}{2} \frac{\mathrm{d}}{\mathrm{d} t}|\nabla u|^{2}+\nu|\Delta u|^{2}=((u \cdot \nabla) u, \Delta u)-(f, \Delta u) .
$$


Bounding the nonlinear term using the Sobolev inequality $|u|_{L^{6}} \leq c|\nabla u|_{L^{2}}$, which is specific to dimension three, we find

$$
|((u \cdot \nabla) u, \Delta u)| \leq|u|_{L^{3}}|\nabla u|_{L^{6}}|\Delta u|_{L^{2}} \leq \frac{c_{1}}{2}|u|_{L^{3}}|\Delta u|_{L^{2}}^{2},
$$
and the forcing term in the obvious fashion

$$
(f, \Delta u) \leq \frac{1}{\nu}|f|^{2}+\frac{\nu}{4}|\Delta u|^{2} .
$$

We then arrive at

$$
\frac{\mathrm{d}}{\mathrm{d} t}|\nabla u|^{2}+\left(3 \nu / 2-c_{1}|u|_{L^{3}}\right)|\Delta u|^{2} \leq \frac{2}{\nu}|f|^{2} .
$$

Assuming that

$$
\left|u_{0}\right|_{L^{3}} \leq \frac{\nu}{4 c_{1}}
$$

we find that on some interval of time $(0, T)$

$$
|u|_{L^{3}} \leq \nu /\left(2 c_{1}\right) .
$$

We then find that

$$
3 \nu / 2-c_{1}|u|_{L^{3}} \geq \nu .
$$

Using the Poincaré inequality, (1.12) becomes on this interval

$$
\frac{\mathrm{d}}{\mathrm{d} t}|\nabla u|^{2}+\frac{\nu}{c_{0}}|\nabla u|^{2} \leq \frac{2}{\nu}|f|_{L^{\infty}\left(L^{2}\right)}^{2},
$$

where $|f|_{L^{\infty}\left(L^{2}\right)}:=\sup _{t \geq 0}|f(t)|_{L^{2}}$. We then have

$$
\frac{\mathrm{d}}{\mathrm{d} t}\left(|\nabla u|^{2} \exp \left(\frac{\nu}{c_{0}} t\right)\right) \leq \exp \left(\frac{\nu}{c_{0}} t\right) \frac{2}{\nu}|f|_{L^{\infty}\left(L^{2}\right)}^{2} .
$$

Integrating from 0 to $t$ (here we change the dummy variable of integration to $s$ ), we deduce that

$$
|\nabla u(t)|^{2} \exp \left(\frac{\nu}{c_{0}} t\right) \leq|\nabla u(0)|^{2}+\frac{2 c_{0}}{\nu^{2}}|f|_{L^{\infty}\left(L^{2}\right)}^{2}\left(\exp \left(\frac{\nu}{c_{0}} t\right)-1\right) .
$$

We obtain

$$
|\nabla u(t)|^{2} \leq|\nabla u(0)|^{2}+\left(2 c_{0} / \nu^{2}\right)|f|_{L^{\infty}\left(L^{2}\right)}^{2}=: K_{1}\left(u_{0}, f ; \nu, \Omega\right) .
$$


60 Using the Sobolev inequality $|u|_{L^{3}}^{2} \leq c|u|_{H^{1 / 2}}^{2} \leq c|u||\nabla u|$, we find that (1.14) and then (1.19) hold for all time provided.

$$
K_{0} K_{1}=\left(\left|u_{0}\right|^{2}+c_{0}|f|_{L^{\infty}\left(H^{-1}\right)}^{2}\right)\left(\left|\nabla u_{0}\right|^{2}+2 c_{0}|f|_{L^{\infty}\left(L^{2}\right)}^{2} / \nu^{2}\right) \leq c_{2}(\Omega) \nu^{4}
$$

where $c_{2}=c_{2}(\Omega)=c / c_{1}^{4}$. It therefore follows that whenever this holds, the $3 \mathrm{~d}$ NSE has a global solution bounded by (1.8) and (1.19).

\section{2. $H^{1}$ estimate for short times}

Let us now recall the uniform $H^{1}$ estimate for short time and arbitrary data. Multiplying (1.1) by $-\Delta u$ in $L^{2}(\Omega)$ and integrating by parts, we find

$$
\frac{1}{2} \frac{\mathrm{d}}{\mathrm{d} t}|\nabla u|^{2}+\nu|\Delta u|^{2}=((u \cdot \nabla) u, \Delta u)-(f, \Delta u) .
$$

Bounding as before the nonlinear term using the Sobolev and interpolation inequalities, we find

$$
\begin{aligned}
\mid((u \cdot \nabla) u), \Delta u)\left.|\leq| u\right|_{L^{6}}|\nabla u|_{L^{3}}|\Delta u|_{L^{2}} & \leq c|\nabla u||\nabla u|_{H^{1 / 2}}|\Delta u| \\
& \leq c|\nabla u|^{3 / 2}|\Delta u|^{3 / 2} \leq \frac{c_{4}}{2 \nu^{3}}|\nabla u|^{6}+\frac{\nu}{2}|\Delta u|^{2},
\end{aligned}
$$

and using the Cauchy-Schwarz inequality for the last term, this gives

$$
\frac{\mathrm{d}}{\mathrm{d} t}|\nabla u|^{2} \leq \frac{c_{4}}{\nu^{3}}|\nabla u|^{6}+\frac{1}{\nu}|f|_{L^{\infty}\left(L^{2}\right)}^{2} .
$$

This implies

$$
\frac{\mathrm{d}}{\mathrm{d} t}\left(|\nabla u|^{2}+F\right) \leq \frac{c_{4}}{\nu^{3}}\left(|\nabla u|^{2}+F\right)^{3},
$$

where $F:=\left(\nu^{2}|f|_{L^{\infty}\left(L^{2}\right)}^{2} / c_{4}\right)^{1 / 3}$. Writing $z(t):=|\nabla u(t)|^{2}+F$ and integrating, we find

$$
\frac{z(t)^{2}}{z(0)^{2}} \leq \frac{1}{1-2 t c_{4} z(0)^{2} / \nu^{3}}
$$

as long as $t<\nu^{3} /\left(2 c_{4} z(0)^{2}\right)$. It is clear from this that our solution will remain bounded, say, $z(t)^{2} \leq 2 z(0)^{2}$, for $0 \leq t \leq \nu^{3} /\left(4 c_{4} z(0)^{2}\right)$.

In view of results in Sections 1.1 and 1.2 we aim to study the $H^{1}$ stability of two different time discretizaion schemes in the following sections; namely, the semi-implicit and the fully implicit Euler scheme. 


\section{Semi-implicit scheme}

Given a fixed $k=\Delta t>0$, we discretise (1.1) in time using the semi-implicit so Euler scheme

$$
\frac{u^{n}-u^{n-1}}{k}+\left(u^{n-1} \cdot \nabla\right) u^{n}+\nabla p=\nu \Delta u^{n}+f^{n},
$$

where $u^{n}$ and $f^{n}$ are approximations such that

$$
u^{n}=u(n \Delta t), \quad f^{n}=\frac{1}{k} \int_{(n-1) k}^{n k} f(t) d t .
$$

For the 2d NSE, this scheme was proved in [4] to be globally stable in $H^{1}$. For the 3d NSE, its stability mirrors that (which is known) of the continuous system, subject to relatively mild timestep restrictions.

We note a few facts that will be useful later on. First, for any $u$ and $v \in L^{2}$,

$$
2(u-v, u)=|u|^{2}-|v|^{2}+|u-v|^{2} .
$$

Next, for $b>0$ and given positive real sequences $\left(x_{n}\right)$ and $\left(r_{n}\right)$ satisfying

$$
(1+b) x_{n} \leq x_{n-1}+r_{n-1},
$$

we have

$$
x_{n} \leq(1+b)^{-n} x_{0}+\frac{1+b}{b} \max _{j} r_{j} .
$$

The $L^{2}$ bound works out essentially as in the continuous case: multiplying (2.1) by $2 k u^{n}$, using (2.3) and noting that $\left(\left(u^{n-1} \cdot \nabla\right) u^{n}, u^{n}\right)=0$, we find

$$
\left|u^{n}\right|^{2}+\left|u^{n}-u^{n-1}\right|^{2}+2 \nu k\left|\nabla u^{n}\right|^{2}=\left|u^{n-1}\right|^{2}+2 k\left(f^{n}, u^{n}\right) .
$$

90 Bounding the forcing term using Cauchy-Schwarz, we obtain

$$
\begin{aligned}
\left|u^{n}\right|^{2}+2 \nu k\left|\nabla u^{n}\right|^{2} & \leq\left|u^{n-1}\right|^{2}+2 k\left(f^{n}, u^{n}\right) \\
& \leq\left|u^{n-1}\right|^{2}+k\left|f^{n}\right|_{H^{-1}}^{2} / \nu+k \nu\left|\nabla u^{n}\right|^{2} .
\end{aligned}
$$

Using the Poincaré inequality, we deduce that

$$
\left(1+\nu k / c_{0}\right)\left|u^{n}\right|^{2} \leq\left|u^{n-1}\right|^{2}+k\left|f^{n}\right|_{H^{-1}}^{2} / \nu .
$$


Integrating this using (2.5), we find for all $n \in\{1,2, \cdots\}$,

$$
\begin{aligned}
\left|u^{n}\right|^{2} & \leq\left|u^{0}\right|^{2}+\frac{c_{0}+\nu k}{\nu^{2}}|f|_{L^{\infty}\left(H^{-1}\right)}^{2} \\
& =K_{0}\left(u_{0}, f ; \nu, \Omega\right)+(k / \nu)|f|_{L^{\infty}\left(H^{-1}\right)}^{2},
\end{aligned}
$$

where $K_{0}$, and $K_{1}$ below, are as in the continuous case. We note that this bound (the rhs of (2.9) ) tends to $K_{0}$ as $k \rightarrow 0$.

\section{1. $H^{1}$ estimate for small data}

Theorem 1. For small data, let the initial data $u_{0} \in H^{1}$, the forcing $f$ and the timestep $k$ satisfy

$$
\left(K_{0}+k|f|_{L^{\infty}\left(H^{-1}\right)}^{2} / \nu\right)\left(K_{1}+2 k|f|_{L^{\infty}\left(L^{2}\right)}^{2} / \nu\right) \leq c_{2}(\Omega) \nu^{4},
$$

where $K_{0}\left(u_{0}, f\right)$ and $K_{1}\left(u_{0}, f\right)$ are as in the continuous case, (1.8) and (1.19). Then $u^{n}$ is bounded in $H^{1}$ as follows,

$$
\left|\nabla u^{n}\right|^{2} \leq K_{1}+(2 k / \nu)|f|_{L^{\infty}\left(L^{2}\right)}^{2} \quad \text { for all } n \geq 0 .
$$
$-2 k \Delta u^{n}$ and using (2.3), we find

$$
\begin{aligned}
\left|\nabla u^{n}\right|^{2} & +\left|\nabla\left(u^{n}-u^{n-1}\right)\right|+2 \nu k\left|\Delta u^{n}\right|^{2} \\
& =\left|\nabla u^{n-1}\right|^{2}-2 k\left(f^{n}, \Delta u^{n}\right)+2 k\left(u^{n-1} \cdot \nabla u^{n}, \Delta u^{n}\right) .
\end{aligned}
$$

Bounding the nonlinear term using the Sobolev inequality,

$$
\left|\left(\left(u^{n-1} \cdot \nabla\right) u^{n}, \Delta u^{n}\right)\right| \leq\left|u^{n-1}\right|_{L^{3}}\left|\nabla u^{n}\right|_{L^{6}}\left|\Delta u^{n}\right|_{L^{2}} \leq c_{1}\left|u^{n-1}\right|_{L^{3}}\left|\Delta u^{n}\right|_{L^{2}}^{2},
$$

and using the Cauchy-Schwarz inequality for the forcing, (2.12) implies

$$
\left|\nabla u^{n}\right|^{2}+\left(3 \nu / 2-c_{1}\left|u^{n-1}\right|_{L^{3}}\right) k\left|\Delta u^{n}\right|^{2} \leq\left|\nabla u^{n-1}\right|^{2}+(2 k / \nu)\left|f^{n}\right|^{2} .
$$

As in (1.14) of Section 1.1] if we now assume that

$$
\left|u^{n-1}\right|_{L^{3}} \leq \nu /\left(2 c_{1}\right)
$$

105

we deduce from (2.14) that

$$
\left(1+\nu k / c_{0}\right)\left|\nabla u^{n}\right|^{2} \leq\left|\nabla u^{n-1}\right|^{2}+2 k\left|f^{n}\right|^{2} / \nu .
$$


As long as (2.15) holds, we can integrate this using (2.5) to get the bound

$$
\begin{aligned}
\left|\nabla u^{n}\right|^{2} & \leq\left|\nabla u^{0}\right|^{2}+\frac{2\left(c_{0}+\nu k\right)}{\nu^{2}}|f|_{L^{\infty}\left(L^{2}\right)}^{2} \\
& \leq K_{1}\left(u_{0}, f ; \nu, \Omega\right)+(2 k / \nu)|f|_{L^{\infty}\left(L^{2}\right)}^{2},
\end{aligned}
$$

which proves (2.11). As in the continuous case, we now use the Sobolev inequality and interpolation inequalities to bound

$$
\left|u^{n-1}\right|_{L^{3}}^{2} \leq c\left|u^{n-1}\right|_{H^{1 / 2}}^{2} \leq c\left|u^{n-1}\right|\left|\nabla u^{n-1}\right| .
$$

The timestep restriction (2.10) then becomes a sufficient condition for (2.15). More explicitly, since (2.10) holds at $n=0$, (2.9) and (2.11) imply that it will hold at $n=1$ and, by induction, for all $n \in\{2, \cdots\}$, i.e. the solution of the scheme (2.1) is bounded uniformly in (discrete) time subject to (2.10). Comparing to (1.20), we note that this condition also depends on the timestep $k$ in addition to $u_{0}$ and $f$. This timestep restriction is however relatively mild compared to that for the fully implicit scheme in $\$ 3$ below.

\section{2. $H^{1}$ estimate for short times}

Theorem 2. For short times, assuming the timestep restriction (2.26), we have

$$
\left|\nabla u^{n}\right|^{2} \leq 2\left|\nabla u^{0}\right|^{2}+F, \quad \text { where } F:=\left(\nu^{2}|f|_{L^{\infty}\left(L^{2}\right)}^{2} / c_{4}\right)^{1 / 3},
$$

for all $n$ such that $n k=t_{n} \leq \nu^{3} /\left(8 c_{4}\left(\left|\nabla u^{0}\right|^{2}+F\right)^{2}\right)$.

Proof. For short-time $H^{1}$ stability, we bound the nonlinear term in (2.12) as in ${ }_{120}(1.22$,

$$
\begin{aligned}
\left|\left(\left(u^{n-1} \cdot \nabla\right) u^{n}, \Delta u^{n}\right)\right| & \leq\left|u^{n-1}\right|_{L^{6}}\left|\nabla u^{n}\right|_{L^{3}}\left|\Delta u^{n}\right|_{L^{2}} \\
& \leq c\left|\nabla u^{n-1}\right|\left|\nabla u^{n}\right|_{H^{1 / 2}}\left|\Delta u^{n}\right| \\
& \leq c\left|\nabla u^{n-1}\right|\left|\nabla u^{n}\right|^{1 / 2}\left|\Delta u^{n}\right|^{3 / 2} \\
& \leq \frac{c_{4}}{2 \nu^{3}}\left|\nabla u^{n-1}\right|^{4}\left|\nabla u^{n}\right|^{2}+\frac{\nu}{2}\left|\Delta u^{n}\right|^{2} .
\end{aligned}
$$

Then, (2.12) becomes

$$
\begin{aligned}
& \left|\nabla u^{n}\right|^{2}+2 \nu k\left|\Delta u^{n}\right|^{2} \\
& \leq\left|\nabla u^{n-1}\right|^{2}-2 k\left(f^{n}, \Delta u^{n}\right)+k \nu\left|\Delta u^{n}\right|^{2}+\frac{k c_{4}}{\nu^{3}}\left|\nabla u^{n-1}\right|^{4}\left|\nabla u^{n}\right|^{2},
\end{aligned}
$$


this implies

$$
\left|\nabla u^{n}\right|^{2} \leq\left|\nabla u^{n-1}\right|^{2}+\frac{c_{4} k}{\nu^{3}}\left|\nabla u^{n-1}\right|^{4}\left|\nabla u^{n}\right|^{2}+\frac{k}{\nu}|f|_{L^{\infty}\left(L^{2}\right)}^{2} .
$$

We can rewrite (2.22) as

$$
\begin{aligned}
& \left(1-\frac{c_{4} k}{\nu^{3}}\left|\nabla u^{n-1}\right|^{4}\right)\left|\nabla u^{n}\right|^{2} \\
& \quad \leq\left(1-\frac{c_{4} k}{\nu^{3}}\left|\nabla u^{n-1}\right|^{4}\right)\left|\nabla u^{n-1}\right|^{2}+\frac{c_{4} k}{\nu^{3}}\left|\nabla u^{n-1}\right|^{6}+\frac{k}{\nu}|f|_{L^{\infty}\left(L^{2}\right)}^{2} .
\end{aligned}
$$

Since we are interested in short times, we assume that $\left|\nabla u^{n-1}\right|^{2} \leq 2\left|\nabla u^{0}\right|^{2}$ for

125

all relevant $n$ and demand that $k$ satisfy

$$
k \leq \frac{\nu^{3}}{8 c_{4}\left|\nabla u^{0}\right|^{4}}
$$

This implies that the brackets in (2.23) are $\geq \frac{1}{2}$, that is

$$
\frac{1}{2} \leq 1-\frac{4 c_{4} k}{\nu^{3}}\left|\nabla u^{0}\right|^{4} \leq 1-\frac{c_{4} k}{\nu^{3}}\left|\nabla u^{n-1}\right|^{4}
$$

For later use we add the extra $F$, which make a stronger condition than (2.24)

$$
k \leq \frac{\nu^{3}}{2 c_{4}\left(2\left|\nabla u^{0}\right|^{2}+F\right)^{2}} .
$$

With this assumption, (2.23) implies

$$
\frac{\left|\nabla u^{n}\right|^{2}-\left|\nabla u^{n-1}\right|^{2}}{k} \leq \frac{2 c_{4}}{\nu^{3}}\left|\nabla u^{n-1}\right|^{6}+\frac{2}{\nu}|f|_{L^{\infty}\left(L^{2}\right)}^{2} .
$$

Unlike its continuous-time analogue (1.23), this difference inequality implies ${ }_{130}\left|\nabla u^{n}\right|<\infty$ for all $n$, although for sufficiently large time $n k$, it (i.e. the bound) grows without bound as $k \rightarrow 0$. This is a well-known pitfall in discretising differential equations in time. To obtain a finite-time bound on $\left|\nabla u^{n}\right|$, we proceed in analogy with (1.24) and define

$$
z_{n}:=\left|\nabla u^{n}\right|^{2}+F
$$

We then get from (2.27)

$$
\frac{z_{n}-z_{n-1}}{k} \leq \frac{2 c_{4}}{\nu^{3}} z_{n-1}^{3}=: g\left(z_{n-1}\right) .
$$


Observe that $g(\zeta)>g(\hat{\zeta})$ whenever $\zeta>\hat{\zeta}$, that is, $g \geq 0$ is a strictly monotone increasing function.

Now let $\zeta_{n}$ be the positive solution of the difference equation,

$$
\frac{\zeta_{n}-\zeta_{n-1}}{k}=g\left(\zeta_{n-1}\right)
$$

and observe that $\zeta_{n} \geq 0$ if $\zeta_{n-1} \geq 0$. Denoting $t_{n}:=n k$, we claim that

$$
\zeta_{n} \leq \zeta\left(t_{n}\right)
$$

where $\zeta(\cdot)$ is the solution of the differential equation

$$
\frac{\mathrm{d} \zeta}{\mathrm{d} t}=g(\zeta) \quad \text { with } \zeta\left(t_{n-1}\right)=\zeta_{n-1} .
$$

To show this, we first note that $\zeta(t)$ is non-decreasing since $g \geq 0$. Then

$$
\zeta\left(t_{n}\right)-\zeta\left(t_{n-1}\right)=\int_{t_{n-1}}^{t_{n}} g(\zeta(t)) \mathrm{d} t \geq \int_{t_{n-1}}^{t_{n}} g\left(\zeta\left(t_{n-1}\right)\right) \mathrm{d} t=k g\left(\zeta_{n-1}\right) .
$$

Thanks to (2.30), we obtain that

$$
\zeta\left(t_{n}\right)-\zeta\left(t_{n-1}\right)=\zeta_{n}-\zeta_{n-1},
$$

and this prove our claim. By induction, taking $\zeta(0)=\zeta_{0}>0$ instead of the initial data in (2.30), we then have $\zeta_{n} \leq \zeta\left(t_{n}\right)$ for all $n \in\{1,2, \cdots\}$. Comparing with the continuous case (1.24) - (1.25), we conclude that $\zeta_{n} \leq \zeta\left(t_{n}\right) \leq 2 \zeta(0)=$ ${ }_{145} 2 \zeta_{0}$ for $n k=t_{n} \leq \nu^{3} /\left(8 c_{4} \zeta_{0}^{2}\right)$.

Taking $\zeta_{0}=z_{0}$, clearly $z_{n} \leq \zeta_{n}$ for all $n \geq 0$. We therefore have

$$
\begin{aligned}
& z_{n}=\left|\nabla u^{n}\right|^{2}+F \leq \zeta_{n} \leq 2 \zeta_{0}=2\left|\nabla u^{0}\right|^{2}+2 F \\
& \Longrightarrow\left|\nabla u^{n}\right|^{2} \leq 2\left|\nabla u^{0}\right|^{2}+F
\end{aligned}
$$

for all $n$ such that $n k=t_{n} \leq \nu^{3} /\left(8 c_{4}\left(\left|\nabla u^{0}\right|^{2}+F\right)^{2}\right)$, which is half as long as the bound in the continuous case.

\section{Fully implicit scheme}

We now consider the fully implicit Euler scheme

$$
\frac{u^{n}-u^{n-1}}{k}+\left(u^{n} \cdot \nabla\right) u^{n}+\nabla p=\nu \Delta u^{n}+f^{n},
$$


with $\nabla \cdot u^{n}=0$ for all $n$ and $u^{0}=u_{0}$. In two space dimensions, uniform boundedness in $H^{1}$ for this scheme was proved in [8]. We can show uniqueness of the numerical scheme (3.1) by restricting $k$ to be small; see Remark 1 below.

The $L^{2}$ bound obtains as before: multiplying (3.1) $)_{1}$ by $2 k u^{n}$ and using (2.3),

$$
\left|u^{n}\right|^{2}+\left|u^{n}-u^{n-1}\right|^{2}+2 \nu k\left|\nabla u^{n}\right|^{2}=\left|u^{n-1}\right|^{2}+2 k\left(f^{n}, u^{n}\right) .
$$

155 implies

$$
\left(1+\nu k / c_{0}\right)\left|u^{n}\right|^{2} \leq\left|u^{n-1}\right|^{2}+k\left|f^{n}\right|_{H^{-1}}^{2} / \nu .
$$

Integrating this using (2.5), we find for all $n \in\{1,2, \cdots\}$,

$$
\begin{aligned}
\left|u^{n}\right|^{2} & \leq\left|u^{0}\right|^{2}+\frac{c_{0}+\nu k}{\nu^{2}}|f|_{L^{\infty}\left(H^{-1}\right)}^{2} \\
& =K_{0}\left(u_{0}, f ; \nu, \Omega\right)+(k / \nu)|f|_{L^{\infty}\left(H^{-1}\right)}^{2} .
\end{aligned}
$$

As before, this bound tends to $K_{0}$ as $k \rightarrow 0$. For later use, we define

$$
\tilde{K}_{0}\left(u_{0}, f ; \nu, \Omega\right):=\left|u_{0}\right|^{2}+\frac{2 c_{0}}{\nu^{2}}|f|_{L^{\infty}\left(H^{-1}\right)}^{2} .
$$

The central ingredient for our main results of the $H^{1}$ stability is the following 160 local-in-time estimate:

Lemma 1. We assume the $L^{2}$ uniform bound (3.4) and that $u^{n-1} \in H^{1}$. Assuming further the timestep restrictions

$$
\begin{aligned}
& K^{(n-1)} \leq \frac{1}{2}\left(\frac{\nu^{3}}{3 c_{4} k}\right)^{1 / 2}, \\
& \left(1+\frac{c_{5}}{\nu^{4}} \tilde{K}_{0} K^{(n-1)}\right) K^{(n-1)}+|f|_{L^{\infty}\left(H^{-1}\right)}^{2} / \nu^{2} \leq\left(\frac{\nu^{3}}{12 c_{4} k}\right)^{1 / 2},
\end{aligned}
$$

where $K^{(n-1)}:=\left|\nabla u^{n-1}\right|^{2}+\left(10 c_{0} / \nu\right)|f|_{L^{\infty}\left(L^{2}\right)}^{2}$, then the solution $u^{n}$ of (3.1) is bounded as $\left|\nabla u^{n}\right|^{2} \leq y_{1}$ where $y_{1}$ is the smallest positive root of the cubic equation (3.10).

Proof. Multiplying (3.1) by $-2 k \Delta u^{n}$, we have

$$
\begin{aligned}
\left|\nabla u^{n}\right|^{2} & +\left|\nabla\left(u^{n}-u^{n-1}\right)\right|^{2}+2 \nu k\left|\Delta u^{n}\right|^{2} \\
& =\left|\nabla u^{n-1}\right|^{2}+2 k\left(\left(u^{n} \cdot \nabla\right) u^{n}, \Delta u^{n}\right)-2 k\left(f^{n}, \Delta u^{n}\right) .
\end{aligned}
$$




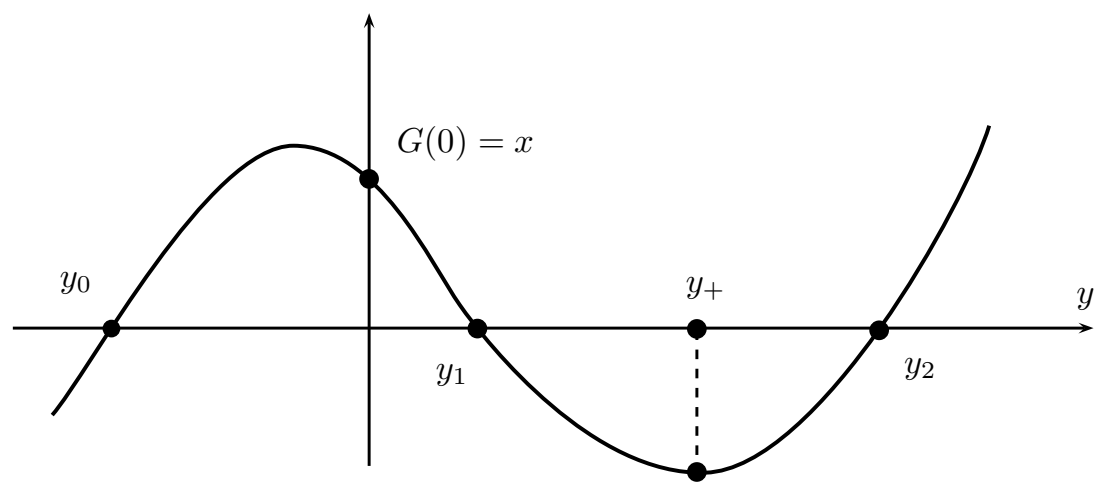

Figure 1: The graph of $G(y)$ in 3.11): $y_{+}$is a local minimum.

Bounding the nonlinear term as we did in (1.22),

$$
2 k\left|\left(\left(u^{n} \cdot \nabla\right) u^{n}, \Delta u^{n}\right)\right| \leq \frac{c_{4} k}{\nu^{3}}\left|\nabla u^{n}\right|^{6}+\nu k\left|\Delta u^{n}\right|^{2},
$$

we find

$$
\begin{aligned}
0 & \leq \frac{c_{4} k}{\nu^{3}}\left|\nabla u^{n}\right|^{6}-\left|\nabla u^{n}\right|^{2}-\frac{\nu k}{2}\left|\Delta u^{n}\right|^{2}+\left|\nabla u^{n-1}\right|^{2}+\frac{2 k}{\nu}\left|f^{n}\right|^{2} \\
\Rightarrow \quad 0 & \leq \frac{c_{4} k}{\nu^{3}}\left|\nabla u^{n}\right|^{6}-\left(1+\frac{\nu k}{2 c_{0}}\right)\left|\nabla u^{n}\right|^{2}+\left|\nabla u^{n-1}\right|^{2}+\frac{2 k}{\nu}|f|_{L^{\infty}\left(L^{2}\right)}^{2} .
\end{aligned}
$$

Let $y:=\left|\nabla u^{n}\right|^{2}, x:=\left|\nabla u^{n-1}\right|^{2}+2 k|f|_{L^{\infty}\left(L^{2}\right)}^{2} / \nu$ and

$$
G(y ; x):=\left(c_{4} k / \nu^{3}\right) y^{3}-\left(1+\nu k /\left(2 c_{0}\right)\right) y+x .
$$

We write $G(y)$ instead of $G(y ; x)$ when there is no risk of confusion. We are of course interested in the solution set of $G(y) \geq 0$.

Under the assumption (3.14) below on the timestep $k$, the graph of the cubic $G$ is (qualitatively) as shown in Figure 1, We note in particular that $G(y)=0$ has a negative root $y_{0}$ and two positive roots $y_{1}$ and $y_{2}$. To verify this, we note the following. First, $G(y) \rightarrow \pm \infty$ as $y \rightarrow \pm \infty$. Next, $G(y)$ has two local extrema,

$$
y_{ \pm}= \pm\left(\frac{\nu^{3}}{3 c_{4} k}\left[1+\frac{\nu k}{2 c_{0}}\right]\right)^{1 / 2}
$$

with $y_{-}<0$ being a local maximum and $y_{+}>0$ a local minimum, as verified 175 
have $G\left(y_{-}\right)>0$. Finally, computing

$$
G\left(y_{+}\right)=-\frac{2}{3}\left(1+\frac{\nu k}{2 c_{0}}\right)\left(\frac{\nu^{3}}{3 c_{4} k}\left[1+\frac{\nu k}{2 c_{0}}\right]\right)^{1 / 2}+x,
$$

we conclude that $G\left(y_{+}\right)<0$ if (this is essentially a restriction on $k$ )

$$
\left|\nabla u^{n-1}\right|^{2}+\frac{2 k}{\nu}|f|_{L^{\infty}\left(L^{2}\right)}^{2}<\frac{2}{3}\left(\frac{\nu^{3}}{3 c_{4} k}\right)^{1 / 2} .
$$

This implies the existence of the two positive roots $y_{1}$ and $y_{2}$ with $y_{1}<y_{+}<y_{2}$.

Now (3.10) implies that $\left|\nabla u^{n}\right|^{2}=y$ lies in the disjoint set $\left[0, y_{1}\right] \cup\left[y_{2}, \infty\right)$. However, $y_{2}>y_{+} \sim k^{-1 / 2}$, which is absurd for small $k$. To prove that $y \notin$ $\left[y_{2}, \infty\right)$, we multiply $(3.1)_{1}$ by $2 k\left(u^{n}-u^{n-1}\right)$ in $L^{2}$ to get

$$
\begin{aligned}
2\left|u^{n}-u^{n-1}\right|^{2} & +\nu k\left|\nabla u^{n}\right|^{2}-\nu k\left|\nabla u^{n-1}\right|^{2}+\nu k\left|\nabla\left(u^{n}-u^{n-1}\right)\right|^{2} \\
& =-2 k\left(\left(u^{n} \cdot \nabla\right) u^{n}, u^{n}-u^{n-1}\right)+2 k\left(f^{n}, u^{n}-u^{n-1}\right)=: I_{1}+I_{2} .
\end{aligned}
$$

Bounding the rhs as

$$
\begin{aligned}
\left|I_{2}\right| & \leq \frac{k}{\nu}\left|f^{n}\right|_{H^{-1}}^{2}+\nu k\left|\nabla\left(u^{n}-u^{n-1}\right)\right|^{2} & & \\
\left|I_{1}\right| & =2 k\left|\left(\left(u^{n} \cdot \nabla\right) u^{n}, u^{n-1}\right)\right| & & \leq 2 k\left|u^{n}\right|_{L^{3}}\left|\nabla u^{n}\right|_{L^{2}}\left|u^{n-1}\right|_{L^{6}} \\
& \leq c k\left|u^{n}\right|_{H^{1 / 2}}\left|\nabla u^{n}\right|\left|\nabla u^{n-1}\right| & & \leq c k\left|u^{n}\right|^{1 / 2}\left|\nabla u^{n}\right|^{3 / 2}\left|\nabla u^{n-1}\right| \\
& \leq \frac{\nu k}{2}\left|\nabla u^{n}\right|^{2}+\frac{c k}{\nu^{3}}\left|u^{n}\right|^{2}\left|\nabla u^{n-1}\right|^{4}, & &
\end{aligned}
$$

and dropping the $2\left|u^{n}-u^{n-1}\right|^{2}$ on the lhs in (3.15), we arrive at

$$
\left|\nabla u^{n}\right|^{2} \leq\left(2+\frac{2 c_{5}}{\nu^{4}}\left|u^{n}\right|^{2}\left|\nabla u^{n-1}\right|^{2}\right)\left|\nabla u^{n-1}\right|^{2}+\frac{2}{\nu^{2}}|f|_{L^{\infty}\left(H^{-1}\right)}^{2} .
$$

If we now assume that (effectively a timestep restriction)

$$
\left(2+\frac{2 c_{5}}{\nu^{4}}\left|u^{n}\right|^{2}\left|\nabla u^{n-1}\right|^{2}\right)\left|\nabla u^{n-1}\right|^{2}+\frac{2}{\nu^{2}}|f|_{L^{\infty}\left(H^{-1}\right)}^{2} \leq\left(\frac{\nu^{3}}{3 c_{4} k}\right)^{1 / 2},
$$

noting that the rhs $<y_{+}<y_{2}$, we can conclude that $\left|\nabla u^{n}\right|^{2}<y_{2}$ and therefore ${ }_{185}\left|\nabla u^{n}\right|^{2} \in\left[0, y_{1}\right]$. This gives us the local $H^{1}$ integrability of the scheme (3.1): if $k$ (is small enough that it) satisfies (3.14) and (3.17), the one-step solution of (3.1) is bounded in $H^{1}$. 
The crucial point which is not immediately obvious is that $y_{1}=\left|\nabla u^{n-1}\right|^{2}+$ $\mathrm{O}(k)$ for small $k$. By estimating the $\mathrm{O}(k)$ more carefully, we obtain our main results.

\section{1. $H^{1}$ estimate for small data}

Theorem 3. For small data, let $u_{0}$ and $f$ be such that

$$
\left|\nabla u_{0}\right|^{2}+\frac{2 c_{0}}{\nu^{2}}|f|_{L^{\infty}\left(L^{2}\right)}^{2} \leq \frac{\nu^{2}}{2 \sqrt{c_{0} c_{4}}}
$$

and let the timestep $k$ satisfy (3.24)-(3.26) below. Then $u^{n}$ is bounded as

$$
\left|\nabla u^{n}\right|^{2} \leq \tilde{K}_{1}\left(u_{0}, f ; \nu, \Omega\right):=\left|\nabla u_{0}\right|^{2}+\frac{10 c_{0}}{\nu^{2}}|f|_{L^{\infty}\left(L^{2}\right)}^{2},
$$

for all $n \in\{0,1, \cdots\}$.

Proof. For small data, we first assume the hypotheses (local in $n$ ) of Lemma 1 . We then derive a more useful explicit bound for $\left|\nabla u^{n-1}\right|^{2}$. We claim that with the assumption (3.18), $\left|\nabla u^{n}\right|^{2} \leq y_{1}$ implies

$$
\left(1+\frac{\nu k}{4 c_{0}}\right)\left|\nabla u^{n}\right|^{2} \leq\left|\nabla u^{n-1}\right|^{2}+\frac{2 k}{\nu}|f|_{L^{\infty}\left(L^{2}\right)}^{2},
$$

where $y_{1}$ is the smallest positive root of the cubic equation (3.10). To prove this, we set $y_{*}:=\left(\left|\nabla u^{n-1}\right|^{2}+2 k|f|_{L^{\infty}\left(L^{2}\right)}^{2} / \nu\right) /\left(1+\nu k /\left(4 c_{0}\right)\right)$ and compute

$$
G\left(y_{*}\right)=y_{*}\left(1+\frac{\nu k}{4 c_{0}}\right)^{-2}\left\{-\frac{\nu k}{4 c_{0}}\left(1+\frac{\nu k}{4 c_{0}}\right)^{2}+\frac{c_{4} k}{\nu^{3}} x^{2}\right\},
$$

where $G(y)$ is as in (3.11) such that

$$
G(y)=\left(c_{4} k / \nu^{3}\right) y^{3}-\left(1+\nu k /\left(2 c_{0}\right)\right) y+x
$$

and $x:=\left|\nabla u^{n-1}\right|^{2}+2 k|f|_{L^{\infty}\left(L^{2}\right)}^{2} / \nu$. Now $G\left(y_{*}\right) \leq 0$ implies that $y_{*} \geq y_{1}$, and the former is true if

$$
-\frac{\nu k}{4 c_{0}}\left(1+\frac{\nu k}{4 c_{0}}\right)^{2}+\frac{c_{4} k}{\nu^{3}} x^{2} \leq 0
$$

200

and this implies

$$
\left|\nabla u^{n-1}\right|^{2}+\frac{2 k}{\nu}|f|_{L^{\infty}\left(L^{2}\right)}^{2}=x \leq \frac{\nu^{2}}{2 \sqrt{c_{0} c_{4}}}
$$


Hence, $\left|\nabla u^{n}\right|^{2} \leq y_{1} \leq y_{*}$ implies (3.20). Then, the claim is proved.

To obtain the uniform bound, we sum (3.20) using (2.5) to find

$$
\left|\nabla u^{n}\right|^{2} \leq\left(1+\frac{\nu k}{4 c_{0}}\right)^{-n}\left|\nabla u^{0}\right|^{2}+\frac{8 c_{0}}{\nu^{2}}|f|_{L^{\infty}\left(L^{2}\right)}^{2}+\frac{2 k}{\nu}|f|_{L^{\infty}\left(L^{2}\right)}^{2} .
$$

Assuming that

$$
k \leq c_{0} / \nu
$$

we can absorb the last term into the penultimate one to obtain (3.19). Consolidating our assumptions, the smallness condition (3.22) is now implied by (3.18), while the timestep restrictions (3.14) and (3.17) can both be satisfied by taking $k$ sufficiently small to satisfy

$$
\begin{aligned}
& \tilde{K}_{1} \leq \frac{1}{2}\left(\frac{\nu^{3}}{3 c_{4} k}\right)^{1 / 2}, \\
& \left(1+\frac{c_{5}}{\nu^{4}} \tilde{K}_{0} \tilde{K}_{1}\right) \tilde{K}_{1}+|f|_{L^{\infty}\left(H^{-1}\right)}^{2} / \nu^{2} \leq\left(\frac{\nu^{3}}{12 c_{4} k}\right)^{1 / 2} .
\end{aligned}
$$

This proves the theorem.

We note that, up to the constant depending only on the domain $\Omega$, the bound (3.19) is the same as that in the continuous case (1.19). In addition, considering $|f|_{H^{-1}}^{2} \leq c_{0}^{*}|f|_{L^{2}}^{2}$ and using Poincaré inequality, we estimate $K_{0}$ in (1.20) such that

$$
K_{0}:=\left|u_{0}\right|^{2}+c_{0}|f|_{L^{\infty}\left(H^{-1}\right)}^{2} \leq c_{0}\left|\nabla u_{0}\right|^{2}+c_{0} c_{0}^{*}|f|_{L^{\infty}\left(L^{2}\right)}^{2} .
$$

Assuming $k \leq\left(c_{0}^{*} \nu\right) / 2$, we deduce from (3.22) that

$$
c_{0}\left(\left|\nabla u_{0}\right|^{2}+c_{0}^{*}|f|_{L^{\infty}\left(L^{2}\right)}^{2}\right) \leq \frac{\nu^{2} \sqrt{c_{0}}}{2 \sqrt{c_{4}}} .
$$

From (3.18) and (3.28), we find, up to the constant depending only on the domain $\Omega$, the same smallness condition as in (1.20).

\section{2. $H^{1}$ estimate for short times}

Theorem 4. For short times, let the timestep $k$ satisfy (3.40), (3.41) and (3.42) below. Then there exists a $t_{f}^{*}=\nu^{3} /\left(8 c_{4} \zeta_{0}^{2}\right)$, as long as $0 \leq n k \leq t_{f}^{*}$ we have

$$
\left|\nabla u^{n}\right|^{2} \leq \tilde{K}_{2}\left(u_{0}, f ; \nu, \Omega\right):=2\left|\nabla u_{0}\right|^{2}+\left(\nu^{2}|f|_{L^{\infty}\left(L^{2}\right)}^{2} / c_{4}\right)^{1 / 3} .
$$


root of a cubic $G\left(y_{1}\right)=0$, the bound $\left|\nabla u^{n}\right|^{2} \leq y_{1}$ is not very convenient, so we compute a more useful bound. Recalling that $x>0$, we consider for some $a>0$

$$
\begin{aligned}
G((1+a k) x ; x) & =x k\left[\frac{c_{4}}{\nu^{3}}(1+a k)^{3} x^{2}-\left(\frac{\nu}{2 c_{0}}+a\right)-\frac{a \nu k}{2 c_{0}}\right] \\
& <x k\left[\frac{c_{4}}{\nu^{3}}(1+a k)^{3} x^{2}-a\right] .
\end{aligned}
$$

Setting $a=2 c_{4} x^{2} / \nu^{3}$, this implies $G((1+a k) x)<0$ if

$$
1+a k \leq 2^{1 / 3} \Leftrightarrow\left(\left|\nabla u^{n-1}\right|^{2}+2 k|f|_{L^{\infty}\left(L^{2}\right)}^{2} / \nu\right)^{2} 2 c_{4} k / \nu^{3} \leq 2^{1 / 3}-1
$$

Assuming this, Lemma 1 then gives us the explicit one-step estimate

$$
\begin{aligned}
\left|\nabla u^{n}\right|^{2} & \leq y_{1} \leq(1+a k) x \\
& =\left|\nabla u^{n-1}\right|^{2}+\frac{2 k}{\nu}|f|_{L^{\infty}\left(L^{2}\right)}^{2}+\frac{2 c_{4} k}{\nu^{3}}\left(\left|\nabla u^{n-1}\right|^{2}+(2 k / \nu)|f|_{L^{\infty}\left(L^{2}\right)}^{2}\right)^{3}
\end{aligned}
$$

220

which we can rewrite as

$$
\frac{\left|\nabla u^{n}\right|^{2}-\left|\nabla u^{n-1}\right|^{2}}{k} \leq \frac{2 c_{4}}{\nu^{3}}\left[\left(\left|\nabla u^{n-1}\right|^{2}+\frac{2 k}{\nu}|f|_{L^{\infty}\left(L^{2}\right)}^{2}\right)^{3}+\frac{\nu^{2}}{c_{4}}|f|_{L^{\infty}\left(L^{2}\right)}^{2}\right] .
$$

To obtain a finite-time bound on $\left|\nabla u^{n}\right|$, we proceed in analogy with (1.24) and define

$$
z_{n}:=\left|\nabla u^{n}\right|^{2}+F \quad \text { where } F^{3}=\frac{2 \nu^{2}}{c_{4}}|f|_{L^{\infty}\left(L^{2}\right)}^{2} .
$$

By expanding both sides, we have

$$
\left(\left|\nabla u^{n-1}\right|^{2}+\frac{2 k}{\nu}|f|_{L^{\infty}\left(L^{2}\right)}^{2}\right)^{3}+\frac{\nu^{2}}{c_{4}}|f|_{L^{\infty}\left(L^{2}\right)}^{2} \leq z_{n-1}^{3},
$$

subject to the timestep restriction

$$
k \leq \frac{\nu^{5 / 3}}{2 c_{4}^{1 / 3}|f|_{L^{\infty}\left(L^{2}\right)}^{4 / 3}} \Rightarrow\left\{\begin{array}{l}
4^{1 / 3} c_{4}^{1 / 3}|f|_{L^{\infty}\left(L^{2}\right)}^{4 / 3} k \leq \nu^{5 / 3} \\
4^{2 / 3} c_{4}^{2 / 3}|f|_{L^{\infty}\left(L^{2}\right)}^{8 / 3} k^{2} \leq \nu^{10 / 3} \\
8 c_{4}|f|_{L^{\infty}\left(L^{2}\right)}^{4} k^{3} \leq \nu^{5}
\end{array}\right.
$$

The time step restriction above can be found by direct computations. Indeed, 
expanding (3.35), we have

$$
\begin{gathered}
\left|\nabla u^{n-1}\right|^{6}+3\left|\nabla u^{n-1}\right|^{4}\left(\frac{2 k}{\nu}|f|_{L^{\infty}\left(L^{2}\right)}^{2}\right)+3\left|\nabla u^{n-1}\right|^{2}\left(\frac{2 k}{\nu}|f|_{L^{\infty}\left(L^{2}\right)}^{2}\right)^{2} \\
+\left(\frac{2 k}{\nu}|f|_{L^{\infty}\left(L^{2}\right)}^{2}\right)^{3}+\frac{\nu^{2}}{c_{4}}|f|_{L^{\infty}\left(L^{2}\right)}^{2} \\
\leq\left|\nabla u^{n-1}\right|^{6}+3\left|\nabla u^{n-1}\right|^{4} F+3\left|\nabla u^{n-1}\right|^{2} F^{2}+F^{3} \\
=\left|\nabla u^{n-1}\right|^{6}+3\left|\nabla u^{n-1}\right|^{4}\left(\frac{2 \nu^{2}}{c_{4}}|f|_{L^{\infty}\left(L^{2}\right)}^{2}\right)^{1 / 3} \\
+3\left|\nabla u^{n-1}\right|^{2}\left(\frac{2 \nu^{2}}{c_{4}}|f|_{L^{\infty}\left(L^{2}\right)}^{2}\right)^{2 / 3}+\frac{2 \nu^{2}}{c_{4}}|f|_{L^{\infty}\left(L^{2}\right)}^{2} .
\end{gathered}
$$

Hence, assuming the three conditions in the brace of (3.36), the inequality (3.37) holds term by term. Then (3.32) implies

$$
\frac{z_{n}-z_{n-1}}{k} \leq \frac{2 c_{4}}{\nu^{3}} z_{n-1}^{3}=: g\left(z_{n-1}\right) .
$$

Arguing as we did in the semi-implicit case [cf. [2.30)-(2.33)], we conclude that ${ }_{230} z_{n} \leq 2 z_{0}$ for all $n \geq 0$ such that

$$
n k=t_{n} \leq \nu^{3} /\left(8 c_{4} \zeta_{0}^{2}\right)=: t_{f}^{*}
$$

This proves the theorem subject to the timestep restrictions, which we collect here. First, (3.6) and (3.7) are implied by

$$
\begin{aligned}
& \tilde{K} \leq \frac{1}{2}\left(\frac{\nu^{3}}{3 c_{4} k}\right)^{1 / 2} \\
& \left(1+\frac{c_{5}}{\nu^{4}} \tilde{K}_{0} \tilde{K}\right) \tilde{K}+|f|_{L^{\infty}\left(H^{-1}\right)}^{2} / \nu^{2} \leq\left(\frac{\nu^{3}}{12 c_{4} k}\right)^{1 / 2},
\end{aligned}
$$

where unlike in Lemma 1 here

$$
\tilde{K}:=2\left|\nabla u^{0}\right|^{2}+2\left(\nu^{2}|f|_{L^{\infty}\left(L^{2}\right)}^{2} / c_{4}\right)^{1 / 3}+\left(10 c_{0} / \nu\right)|f|_{L^{\infty}\left(L^{2}\right)}^{2}
$$

Next, (3.36) is good as it stands. Finally, using (3.36) to handle the $k$ inside the bracket, (3.31) is implied by

$$
\left(2\left|\nabla u^{0}\right|^{2}+\frac{\left(1+2^{1 / 3}\right) \nu^{2 / 3}|f|_{L^{\infty}\left(L^{2}\right)}^{2 / 3}}{c_{4}^{1 / 3}}\right)^{2} \leq \frac{\left(2^{1 / 3}-1\right) \nu^{3}}{2 c_{4} k} .
$$

This proves the short-time case. 
The time bound $t_{f}^{*}$ is essentially that of the continuous case (smaller by a factor $\frac{1}{2}$ which can be improved to $1-\varepsilon$ with some work and more restrictions on $k)$.

Remark 1. For the issue of uniqueness of solution for the numerical scheme (3.1), we consider two solutions of (3.1), $u_{1}^{n}, u_{2}^{n}$, and $u^{n}=u_{1}^{n}-u_{2}^{n}$ :

$$
\begin{aligned}
& \frac{u_{1}^{n}-u^{n-1}}{k}-\nu \Delta u_{1}^{n}+u_{1}^{n} \cdot \nabla u_{1}^{n}=f^{n}, \\
& \frac{u_{2}^{n}-u^{n-1}}{k}-\nu \Delta u_{2}^{n}+u_{2}^{n} \cdot \nabla u_{2}^{n}=f^{n} .
\end{aligned}
$$

$$
\left|u^{n}\right|^{2}+k \nu\left\|u^{n}\right\|^{2}=-k b\left(u^{n}, u_{2}^{n}, u^{n}\right) .
$$

For the trilinear term, we use the $L^{4}-L^{2}-L^{4}$ estimate, and we find, for some constant $c_{5}$ :

$$
\begin{aligned}
k\left|b\left(u^{n}, u_{2}^{n}, u^{n}\right)\right| & \leq \text { (see Lemma 3.5, Chapter 3 in [u] }) \\
& \leq c_{5} k\left|u^{n}\right|^{1 / 4}\left\|u^{n}\right\|^{3 / 4}\left\|u_{2}^{n}\right\|\left|u^{n}\right|^{1 / 4}\left\|u^{n}\right\|^{3 / 4} .
\end{aligned}
$$

By Theorems 可and 4 above, we have

$$
\left\|u_{2}^{n}\right\|^{2} \leq \max \left(\tilde{K}_{1}, \tilde{K}_{2}\right)
$$

where $\tilde{K}_{1}$ and $\tilde{K}_{2}$ are as in (3.19) and (3.29), respectively; note that here we are writing that $\left|f^{n}\right|_{L^{2}} \leq|f|_{L^{\infty}\left(L^{2}\right)}$. By Young's inequality, we deduce from (3.46) that

$$
\begin{aligned}
k\left|b\left(u^{n}, u_{2}^{n}, u^{n}\right)\right| & \leq\left[c_{5}^{2} \max \left(\tilde{K}_{1}, \tilde{K}_{2}\right)\right]^{1 / 2} k\left|u^{n}\right|^{1 / 2}\left\|u^{n}\right\|^{3 / 2} \\
& \leq \frac{1}{4}\left|u^{n}\right|^{2}+\tilde{K}_{3} k^{4 / 3}\left\|u^{n}\right\|^{2}
\end{aligned}
$$

where

$$
\tilde{K}_{3}:=\tilde{K}_{3}\left(u_{0}, f ; \nu, \Omega\right)=\frac{3}{4}\left[c_{5}^{2} \max \left(\tilde{K}_{1}, \tilde{K}_{2}\right)\right]^{2 / 3} .
$$

We then find that

$$
\frac{3}{4}\left|u^{n}\right|^{2}+k\left(\nu-\tilde{K}_{3} k^{1 / 3}\right)\left\|u^{n}\right\|^{2} \leq 0 .
$$


Assuming that $k$ is small, so that

$$
k^{1 / 3} \leq \nu \tilde{K}_{3}^{-1}
$$

(3.49) gives $u^{n}=0$ and the uniqueness of solution for the scheme (3.1). Note 250 that the condition (3.50) on the mesh $k$ is valid for all steps $n$, after observing that $\left|f^{n}\right|_{L^{2}} \leq|f|_{L^{\infty}\left(L^{2}\right)}$.

\section{Acknowledgement}

This work was supported in part by NSF Grant DMS 1206438 and by the Research Fund of Indiana University.

\section{References}

[1] P. Constantin, C. Foias, Navier-Stokes equations, Chicago Lectures in Mathematics, University of Chicago Press, Chicago, IL, 1988.

[2] T. Geveci, On the convergence of a time discretization scheme for the NavierStokes equations, Math. Comp. 53 (187) (1989) 43-53.

URL http://dx.doi.org/10.2307/2008348

[3] S. Gottlieb, F. Tone, C. Wang, X. Wang, D. Wirosoetisno, Long time stability of a classical efficient scheme for two-dimensional Navier-Stokes equations, SIAM J. Numer. Anal. 50 (1) (2012) 126-150.

URL http://dx.doi.org/10.1137/110834901

[4] N. Ju, On the global stability of a temporal discretization scheme for the Navier-Stokes equations, IMA J. Numer. Anal. 22 (4) (2002) 577-597.

URL http://dx.doi .org/10.1093/imanum/22.4.577

[5] M. Marion, R. Temam, Navier-Stokes equations: theory and approximation, in: Handbook of numerical analysis, Vol. VI, Handb. Numer. Anal., VI, North-Holland, Amsterdam, 1998, pp. 503-688. 
[6] J. Shen, Long time stability and convergence for fully discrete nonlinear Galerkin methods, Appl. Anal. 38 (4) (1990) 201-229.

URL http://dx.doi.org/10.1080/00036819008839963

[7] R. Temam, Navier-Stokes equations, AMS Chelsea Publishing, Providence, RI, 2001, theory and numerical analysis, Reprint of the 1984 edition.

[8] F. Tone, D. Wirosoetisno, On the long-time stability of the implicit Euler scheme for the two-dimensional Navier-Stokes equations, SIAM J. Numer. Anal. 44 (1) (2006) 29-40.

URL http://dx.doi .org/10.1137/040618527

[9] X. Wang, An efficient second order in time scheme for approximating long time statistical properties of the two dimensional Navier-Stokes equations, Numer. Math. 121 (4) (2012) 753-779.

URL http://dx.doi .org/10.1007/s00211-012-0450-3 